paranoid individuals will show normal or high selfesteem on overt measures, whereas covert measures will show hidden feelings of low self-esteem.

Methods: The present study used a new methodology that has been widely used in investigations of implicit attitudes, the Implicit Association Test (IAT), to assess covert self-esteem and to test the above prediction. Overt self-esteem was assessed using the Rosenberg Self-Esteem Scale and an adjective self-relevance ratings measure. These measures were administered to 10 patients with acute persecutory delusions, 10 patients with persecutory delusions in remission and 19 matched healthy control participants.

Results: Patients with acute persecutory delusions were found to have lower covert self-esteem (as assessed using the IAT) than healthy controls and patients with remitted persecutory delusions. On the two measures of overt self-esteem, however, the persecutory deluded group did not differ significantly from the other groups once the effects of comorbid depression had been taken into account.

Conclusions: The results of the present study are consistent with a model of persecutory delusions as serving a defensive function. As such, they are consistent with a psychotherapeutic approach to what are perhaps the most frequently observed symptoms of schizophrenia.

\section{Impaired psychosocial functioning in bipolar disorder: the relative contributions of inter-episodic symptoms and illness episodes}

\section{P Mitchell'1, , G Malhi' ${ }^{2,3,4}$, J Ball', A Johnston', D Hadzi-Pavlovic' ${ }^{1}$, J Corry ${ }^{1}$}

'School of Psychiatry, University of New South Wales; ${ }^{2}$ The Black Dog Institute; ${ }^{3}$ Academic Discipline of Psychological Medicine, Northern Clinical School, The University of Sydney; and "Prince of Wales Medical Research Institute, Sydney, Australia

Background: This study examines the relationship between the presence of inter-episodic symptomatology, the frequency of depressed and (hypo)manic episodes, and impairment across eight different domains of functioning. Method: Patients $(n=217)$ attending a tertiary referral center for the assessment of bipolar disorder, who met DSM-IV criteria for BP I or BP II disorder, were included in the analysis. Data were collected on 10 common inter-episodic symptoms reflecting both mood and anxiety, and the frequency of depressive and (hypo)manic episodes. Impairment in the following functional domains was also determined: occupation, financial affairs, self-care, housekeeping, intimate and nonintimate relationships, and parenting. Logistic regression analyses were conducted to examine the number of inter-episodic symptoms reported and the frequency of frank bipolar disorder episodes as predictors of impairment in eight domains of psychosocial functioning.

Results: Psychosocial functional impairment and inter-episodic symptoms were highly prevalent in the sample. The number of inter-episodic symptoms was significantly associated with relationship impairment and employment status, while the frequency of (hypo)manic episodes was associated with impairment in housekeeping and parenting roles. There was no apparent relationship between frequency of depressive episodes and functional capacity.

Conclusions: Both the 'dosage' of inter-episodic (subsyndromal) symptoms and the frequency of (hypo)manic episodes appear to be significant and independent determinants of psychosocial functioning. It is possible that certain aspects of bipolar disorder have a greater impact on some functional domains. Possible methodological reasons for the failure to show a relationship with the frequency of depressive episodes are discussed.

\section{A factor analytic study in bipolar depression, and response to lamotrigine}

\author{
P Mitchell ${ }^{12}$, G Malhi' ${ }^{2,3,4}$, D Hadzi-Pavlovic ${ }^{1}$ \\ 'School of Psychiatry, University of New South Wales; ${ }^{2}$ The Black Dog Institute; \\ ${ }^{3}$ Academic Discipline of Psychological Medicine, Northern Clinical School, \\ The University of Sydney; and APrince of Wales Medical Research Institute, \\ Sydney, Australia
}

Background: This study aimed to identify and compare factors of a 31-item version of the HDRS (HDRS-31) in large samples of patients with bipolar and unipolar depression, then examine for any responsiveness of such factors to the anticonvulsant agent lamotrigine in the bipolar depressed sample.

Methods: This multivariate analytical study was performed on two large depressed samples (one bipolar and the other unipolar) that had been recruited for separate double-blind placebo-controlled trials of lamotrigine. Both studies had very similar designs and assessment tools, the major measures being the MADRS and HDRS-31. To identify the constructs underlying the scale, exploratory factor analyses were applied to the HDRS-31. Treatment responsiveness in the bipolar depressed sample - as indicated by improvement in the total MADRS and HDRS-31, as well as any HDRS factors - was examined using both a mixed-effects analysis and individual time-point $t$-tests.

Results: Seven factors of the HDRS-31 were identified: I - 'depressive cognitions', II - 'psychomotor retardation', III - 'insomnia', IV - 'hypersomnia', 
V - 'appetite and weight change', VI - 'anxiety' and VII - 'anergia'. A significant therapeutic effect of lamotrigine in bipolar depression was found using the 'depressive cognitions' factor (from week 3) and 'psychomotor retardation' (from week 4).

Conclusions: This study has identified seven factors of the HDRS in a large sample of patients with bipolar depression. It suggests that the major effect of lamotrigine in bipolar depression is primarily upon central depressive cognitions and psychomotor disturbance.

\section{Evaluation of MoodGYM with an adolescent population}

\section{A Neil, H Christensen, K Griffiths}

Centre for Mental Health Research, The Australian National University, Canberra, Australia

Background: MoodGYM is an interactive web-based program designed to prevent and decrease symptoms of anxiety and depression. Based on cognitive behaviour therapy, the MoodGYM program aims to change dysfunctional thoughts and improve problem solving, relaxation and interpersonal skills. Although much research has been conducted on the effectiveness of MoodGYM with an adult population, with very positive results, little research has been conducted on the adolescent and young adult population for which the program was designed. The current study therefore aims to evaluate the effectiveness of the MoodGYM program in reducing adolescents' symptoms of anxiety and depression.

Methods: Twenty-nine schools from across Australia were involved in the current study, with 1450 students aged 13-17 years consenting to participate. Each school was randomly assigned to the experimental or control group based on their location (city or rural) and funding (government or private). All participating students completed the first self-report questionnaire the week before students in the experimental condition began the MoodGYM program. The MoodGYM program was delivered to students over a 5-week period. All participating students completed a second and third self-report questionnaire 1 and 6 months after the completion of the MoodGYM program.

Results: The current paper will present preliminary results from the first two questionnaires. Pre- and posttest comparisons will be made for the measures of anxiety and depression.

Conclusion: Findings from the current study will help to inform schools about the usefulness of a self-guided approach to learning about mental health using the Internet.

\section{Visual field semantic priming and relationship with thought disorder}

\author{
E Neill', M Collins'², S Collinson ${ }^{3}$, S Rossell'
}

'Mental Health Research Institute of Victoria, Melbourne, Australia; ${ }^{2}$ Murdoch

University, Perth, Australia; and ${ }^{3}$ Singapore University, Singapore

Background: The semantic priming technique can be used to explore language deficits in individuals with thought disorder. Research suggests that thought disorder may reflect a difficulty in inhibition or an increase in spreading activation in one or both of the hemispheres in response to language-based information. Currently, little research has been done in this area and the research that is available is methodologically flawed.

Methods: A lateralized version of a semantic priming task was used to examine the role of each hemisphere in processing language and to examine how the two hemispheres synchronize information. As in standard semantic priming task, reaction times to related and unrelated word pairs were contrasted. Novelly, to this experiment, word pairs were presented solely to the left (LL) or right (RR) visual field, or across visual fields; left-right (LR) or right-left (RL). Participants included 21 healthy controls and 42 participants with a diagnosis of schizophrenia (21 with thought disorder, 21 without). Repeated-measure ANOVAs were used to compare stimulus type (related vs. unrelated), visual field (LL, RR, LR, RL) and group.

Results: Preliminary analyses suggest that there are significant differences in priming across visual fields between healthy controls and participants with schizophrenia. There were no significant differences between the two psychiatric groups.

Conclusions: These data suggest that participants with schizophrenia have difficulty when performing a task where the two hemispheres must synchronize information. However, this pattern this does not seem to be causal for thought disorder.

\section{When things are not as they seem: detecting first-episode psychosis upon referral to ultrahigh-risk ('prodromal') clinics}

\section{B Nelson', A Yung ${ }^{2}$ \\ 'ORYGEN Research Centre; and 'Department of Psychiatry, The University of Melbourne, Melbourne, Australia}

Background: There are two main targets for intervention during the prodromal phase: 1) current symptoms, behaviour or disability and 2) prevention of onset of frank psychotic disorder. The current presentation examines a 'third' function of 'ultrahigh-risk' (UHR) 